\title{
Information Domain Analysis of Respiratory Sinus Arrhythmia Mechanisms
}

\author{
J. KROHOVA ${ }^{1,2}$, B. CZIPPELOVA ${ }^{1,2}$, Z. TURIANIKOVA ${ }^{1,2}$, Z. LAZAROVA $^{1}$, R. WISZT $^{1,2}$, \\ M. JAVORKA ${ }^{1,2}$ L. FAES ${ }^{3,4}$ \\ ${ }^{1}$ Department of Physiology, Jessenius Faculty of Medicine in Martin, Comenius University in \\ Bratislava, Martin, Slovakia, ${ }^{2}$ Biomedical Center Martin, Jessenius Faculty of Medicine in Martin, \\ Comenius University in Bratislava, Martin, Slovakia, ${ }^{3}$ Department of Energy, Information \\ engineering and Mathematical models (DEIM), University of Palermo, Italy, ${ }^{4}$ BIOtech Center of \\ the Department of Industrial Engineering, University of Trento, Italy
}

Received March 23, 2018

Accepted September 12, 2018

\begin{abstract}
Summary
Ventilation related heart rate oscillations - respiratory sinus arrhythmia (RSA) - originate in human from several mechanisms. Two most important of them - the central mechanism (direct communication between respiratory and cardiomotor centers), and the peripheral mechanism (ventilation-associated blood pressure changes transferred to heart rate via baroreflex) have been described in previous studies. The major aim of this study was to compare the importance of these mechanisms in the generation of RSA non-invasively during various states by quantifying the strength of the directed interactions between heart rate, systolic blood pressure and respiratory volume signals. Seventy-eight healthy volunteers (32 male, age range: 16.02-25.77 years, median age: 18.57 years) participated in this study. The strength of mutual interconnections among the spontaneous beat-to-beat oscillations of systolic blood pressure (SBP), R-R interval (RR signal) and respiration (volume changes RESP signal) was quantified during supine rest, orthostatic challenge (head-up tilt, HUT) and cognitive load (mental arithmetics, MA) using bivariate and trivariate measures of cardio-respiratory information transfer to separate baroreflex and nonbaroreflex (central) mechanisms. Our results indicate that both basic mechanisms take part in RSA generation in the intact cardiorespiratory control of human subjects. During orthostatic and mental challenges baroreflex based peripheral mechanism becomes more important.
\end{abstract}

\section{Key words}

Respiratory sinus arrhythmia - Head-up tilt - Baroreflex • Information measures $\bullet$ Cardio-respiratory coupling

\section{Corresponding author}

J. Krohova, Department of Physiology Jessenius Faculty of Medicine in Martin, Comenius University in Bratislava, Mala Hora 4C, 03601 Martin, Slovakia. E-mail: Jana.Krohova@jfmed.uniba.sk

\section{Introduction}

Oscillations occurring at the respiratory frequency are the most well-known component of spontaneous heart rate variability. This phenomenon, termed respiratory sinus arrhythmia (RSA), was firstly described by Ludwig (1847). Since then, numerous studies have been trying to find out the mechanisms underlying this complex phenomenon. During inspiration, the RR interval of the ECG shortens as a consequence of the temporary inhibition of the nucleus ambiguus (cardio-inhibitory center), which causes a reduction of vagal output to the sinoatrial node. On the contrary, expiration activates the cells in the nucleus ambiguus prolonging the RR interval (Grossman and Taylor 2007, Larsen et al. 2010, Mortola et al. 2016).

The activation or inhibition of neurons in cardioinhibitory center is mediated via direct and indirect pathways. The direct pathway (central mechanism) 
reflects the spreading of the neural activity from the respiratory to the cardio-inhibitory centers (Mortola et al. 2016). During breathing, various cardiovascular and respiratory parameters (e.g. intrathoracic pressure, venous return, systemic blood pressure, $\mathrm{pH}$, partial pressure of blood gases $-\mathrm{pCO}_{2}$ and $\mathrm{pO}_{2}$, etc.) oscillate at the rhythm of breathing (Saul et al. 1991). These oscillations have a potential to be transferred to RSA via reflex mechanisms creating indirect pathways (peripheral mechanisms of RSA) (Larsen et al. 2010, Mortola et al. 2016). It has been shown that the transfer of blood pressure oscillations associated with ventilation via baroreflex to RSA is a dominant peripheral mechanism in the generation of RSA in humans (Piepoli et al. 1997, Zhang et al. 2002, Eckberg 2009, Draghici and Taylor 2016).

While previous studies supported the involvement of either the central or the peripheral mechanism in the generation of RSA (Taha et al. 1995, Schäfer et al. 1998), more recent investigations demonstrated that RSA could result from the combined involvement of several mechanisms (Larsen et al. 2010). Studies focused on the analysis of the relative contribution of central and peripheral (baroreflex) mechanisms to the generation of RSA during different states have been performed only rarely, and usually in a small number of subjects and using simple protocols that included only two physiological states (Piepoli et al. 1997, Faes et al. 2011, Porta et al. 2012a, Porta et al. 2012b). A study on a larger sample of healthy subjects focused on the comparison of the importance of the central vs. peripheral mechanisms of RSA during different states is still missing (Berntson et al. 1993, Larsen et al. 2010).

The recent introduction of information-theoretic analysis tools allowing the inference of direct/indirect mutual interactions between biological signals (Faes et al. 2011, Faes et al. 2012) has opened the possibility to assess the relative contribution of mechanisms operating along different pathways to the dynamics of a target physiological variable. The present study aims at exploiting these tools to noninvasively estimate the contribution of central and peripheral mechanisms to the generation of RSA. We hypothesize that the importance of these two major mechanisms of RSA generation varies during different states (rest, stress). Accordingly, we quantify the strength of the mutual relations among heart rate, systolic blood pressure and respiratory volume signals in a large group of healthy subjects studied during orthostatic test and cognitive load (mental arithmetics).

\section{Methods}

\section{Subjects and experimental protocol}

Seventy-eight healthy volunteers (32 male, age range: $16.02-25.77$ years, median age: 18.57 years) were recruited for this study. Subjects were instructed not to use substances influencing autonomic nervous system or cardiovascular system activity. Female subjects were examined in the proliferative phase $\left(6^{\text {th }}-13^{\text {th }}\right.$ day $)$ of the menstrual cycle. All procedures were approved by Ethical Committee of the Jessenius Faculty of Medicine, Comenius University and all participants signed a written informed consent. During recordings, the volunteers were asked to avoid disturbing movements and speaking. They were positioned on the tilt table with the feet in contact with the footboard at the end of the table and restraining strap was secured at the thigh level to provide subject support and safety. The study protocol consisted of five phases: supine rest (15 min); head-up tilt (HUT, during which the subject was tilted to 45 degrees on the motor driven tilt table for $8 \mathrm{~min}$ to evoke mild orthostatic stress); first supine recovery in the resting supine position (10 min); and mental arithmetic task (MA) in the supine position for $(6 \mathrm{~min})$, followed by the second supine recovery in the resting supine position (10 min). During the MA task the subject was instructed to sum up threedigit numbers (until one-digit number was reached) displayed on the ceiling of the examination room by a data projector. After deciding if the final one-digit number was odd or even, the subject had to click by computer mouse on the corresponding push button projected on the ceiling. During the MA task the subject was disturbed by the rhythmic sound of a metronome and instructed to perform the task as quickly as possible with a minimal error rate.

\section{Data acquisition and analysis}

The acquired signals included the continuous finger arterial blood pressure recorded non-invasively by the photoplethysmographic volume-clamp method (Finometer Pro, FMS, Netherlands), the electrocardiogram (horizontal bipolar thoracic lead; CardioFax ECG-9620, NihonKohden, Japan) and the respiratory volume recorded by respiratory inductive plethysmography using two (thoracic and abdominal) elastic bands (RespiTrace, NIMS, USA). All signals were digitized at 1,000 Hz. From these signals, the following 
beat-to-beat time series were measured: the heart period (R-R interval) was calculated as the temporal distance between two consecutive $\mathrm{R}$ waves; the systolic blood pressure (SBP) was taken as the maximum value of the arterial blood pressure waveform following each $\mathrm{R}$ wave; and the respiration value (RESP) was taken as the sample of the measured volume changes sampled at the onset of each $\mathrm{R}$ wave. All signals were linearly detrended to avoid the effect of slow trends in the signals on the data analysis.

To avoid transient changes, the following segments of 300 beats were extracted from the original recordings for data analysis: for the first phase the analysed segment started $8 \mathrm{~min}$ after the beginning of this phase, for the second phase $3 \mathrm{~min}$ after the change of the position, for the third phase $7 \mathrm{~min}$ before starting the MA task, for the fourth phase 2 min after starting of this phase and for the fifth phase 2 min after completing the MA task.

\section{Causal coupling analysis}

The strength of the mutual interconnections between the time series of RESP, SBP and RR was quantified in the information domain by computing causal coupling indexes based on the model-free entropy estimators proposed in Faes et al. (2011) and Faes et al. (2012). We refer to Faes et al. (2011), Faes et al. (2012) and Javorka et al. (2017) for the methodological details. Here, we first calculated the causal coupling indexes using a bivariate approach involving exclusively the RESP and RR time series. The resulting index of causal coupling from RESP to RR is regarded as an overall measure of coupling (regardless of the direct or indirect influence of RESP on RR) and quantifies the amount of information carried by the present value of the target signal (RR) which can be explained by the past of the source signal (RESP). Moreover, in order to distinguish between direct and indirect influences of RESP on RR, we calculated the causal coupling indexes using a trivariate approach which considers all three recorded signals and quantifies how useful is the source signal (RESP) in forecasting the target signal (RR), provided that third signal (SBP) is known. Then, if the causal coupling index calculated using the trivariate analysis is equal to the index calculated using bivariate analysis, i.e. the source signal provides a given amount of information explaining the target signal and the third signal does not provide any additional information on the oscillations in the target signal, the interaction from source to target is direct without an involvement of the third signal as mediator. In this case a prevalently direct influence of the source on the target is assumed. On the other hand, if the causal coupling indexes calculated through trivariate analysis are markedly lower than the indexes calculated through bivariate analysis, the source signal provides no further information explaining the target signal if we already know the third one. In such a case the influence of the source on the target is more indirect as it is mediated mostly by the third signal.

In accordance with the above description, to compare the importance of the central (direct influence of ventilation on heart rate) vs. peripheral mechanism (baroreflex mediated) we assessed if the direct influence $\mathrm{RESP} \rightarrow \mathrm{RR}$ is more important compared to indirect connection $\mathrm{RESP} \rightarrow \mathrm{SBP} \rightarrow \mathrm{RR}$. Therefore we calculated the difference $(\Delta)$ between the bivariate and trivariate coupling indexes as a measure of a degree of indirectionality, i.e. a higher difference indicates a more indirect connection.

\section{Statistical analysis}

The statistical comparison between the magnitude of the coupling index computed along the RESP to RR directions (for bivariate and trivariate approaches) for a given phase of the experiment, or between two subsequent phases for a given index (bivariate or trivariate), was performed through the Wilcoxon signed-rank test. All results were considered statistically significant at a $\mathrm{P}<0.05$. The statistical analysis was performed using SYSTAT 13 (Systat Software Inc., USA).

\section{Results}

Figure 1 shows the distribution of the causal coupling indexes computed across subjects during five phases of the protocol using the bivariate (Fig. 1a) and trivariate (Fig. 1b) approaches. In Figure 1c the difference (delta values) between bivariate and trivariate causal coupling indexes is shown, calculated for each subject as bivariate coupling index minus trivariate coupling index.

The causal coupling in the direction from RESP to RR was significantly higher than the coupling from RR to RESP during the whole protocol using both the bivariate approach $(\mathrm{P} \leq 0.0001)$ and the trivariate approach $(\mathrm{P} \leq 0.0014$, results not shown). Moreover, no significant change in the causal coupling from RESP to RR across 
all phases was found using the bivariate approach $(0.0821 \leq \mathrm{P} \leq 0.9999$, Fig. 1a). Figure $1 \mathrm{~b}$ shows that the trivariate causal coupling from RESP to RR decreased during both HUT (in comparison with supine rest $(\mathrm{P}=0.0297)$ and first supine recovery $(\mathrm{P}=0.0236))$ and MA (compared to a preceding supine recovery -
$\mathrm{P}=0.0382$ ). The difference between bivariate and trivariate coupling indexes along the direction from RESP to $\mathrm{RR}$ is shown in Fig. 1c. The difference increased during HUT in comparison with supine rest $(\mathrm{P}<0.0001)$ and with the following supine recovery $(\mathrm{P}=0.0003)$. (a)

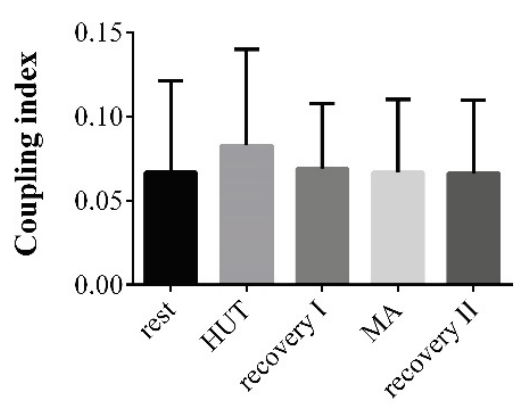

(b)

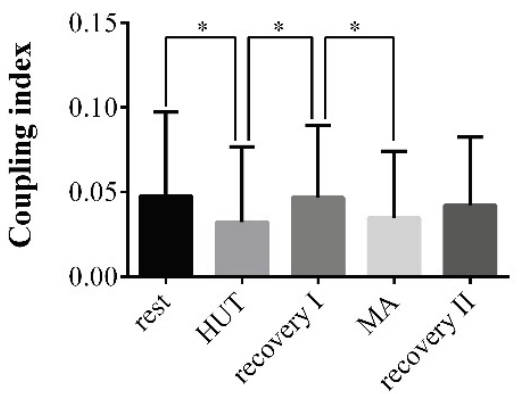

(c)

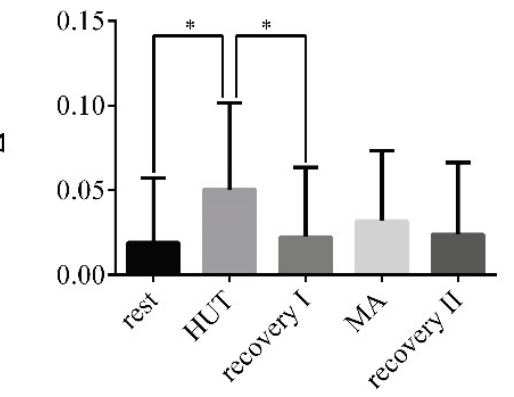

Fig. 1. Causal coupling indexes measured along the direction of the interaction from RESP to RR. The bar plots present the values as mean + SD of the causal coupling in five phases of the protocol (rest, HUT, recovery I, MA, recovery II) using the bivariate approach (a), trivariate approach (b) and the difference between these two indexes is shown in (c). * denotes statistically significant difference between two neighboring phases of the protocol.

\section{Discussion}

RSA - being mediated predominantly by the parasympathetic nervous system - is widely accepted as an indicator of the healthy cardiovascular control at rest (Grossman and Taylor 2007, Larsen et al. 2010, Ben-Tal et al. 2014). A decrease of RSA magnitude is associated with many pathological states, including myocardial infarction (Fei et al. 1996a), and chronic heart failure (Fei et al. 1996b) but it decreases also during challenges leading to a decreased parasympathetic and/or increased sympathetic activity (Allen and Crowell 1989, Herbert et al. 2010, Mortola et al. 2016).

Since RSA was documented for the first time, numerous studies have been performed to reveal basic mechanisms of its origin. Several studies used animal models (Anrep et al. 1936, Ainsworth et al. 1992), or employed pharmacological intervention (atropine, propranolol, trimethaphan) or other manipulations (e.g. voluntary apnea, neck suction, Valsalva maneuver) to describe RSA mechanisms in humans (Saul et al. 1991, Piepoli et al. 1997, Zhang et al. 2002). In general, previous studies demonstrated the involvement of both central and peripheral mechanisms in the generation of RSA, but the relative contribution of these basic mechanisms during different physiological states in human is still not well described (Berntson et al. 1993, Larsen et al. 2010).

The relative role of two basic mechanisms of RSA generation was analysed in healthy human only rarely. The experimental study by Piepoli et al. (1997) in conscious humans (13 healthy men volunteers aged $27.5 \pm 5.9$ years) tried to establish the role of peripheral (baroreflex mediated) and central mechanisms using apnea and the neck suction to separate these mechanisms. The study reported that during normal respiration in sitting position baroreflex is the major mechanism of RSA generation. Recent development of the signal analysis tools aimed to quantify a strength of directed (causal) interactions between oscillations enabled to noninvasively get insight to the mechanisms behind the cardiovascular oscillations origin. Employing the causal analysis of the RR interval and SBP oscillations authors found an increased involvement of baroreflex-mediated mechanism during passive orthostasis (Nollo et al. 2002, Nollo et al. 2005, Porta et al. 2011). However, these studies did not performed trivariate analysis and therefore failed to distinguish between direct and indirect mechanisms of signals interconnections. In the following studies involving trivariate analysis with $\mathrm{SBP}, \mathrm{RR}$ and respiratory signals (Faes et al. 2011, Porta et al. 2012a, Porta et al. 2012b), authors demonstrated a stronger 
influence of central mechanism during supine rest and predominant baroreflex-mediated mechanism during HUT.

We have used recently developed mathematical tools based on information theory to analyze cardiovascular oscillations recorded noninvasively together with the respiratory volume signal. Using bivariate and trivariate analysis, we were able to quantify the strength of mutual interconnections and to distinguish between direct and indirect influence, and thus to compare the involvement of central and peripheral mechanisms of RSA generation during different states (in our study: supine rest, HUT, MA).

To better explain our approach we consider here two extreme cases - one with only direct influence of RESP on RR, the second with only indirect influence $\mathrm{RESP} \rightarrow \mathrm{SBP} \rightarrow \mathrm{RR}$. Firstly, a hypothetical finding that the causal coupling index calculated through trivariate analysis (an interaction from RESP to RR independent of $\mathrm{SBP}$ ) is equal to the index calculated through bivariate analysis (overall interaction from RESP to RR) indicates that SBP does not mediate the effects of RESP on the RR oscillations. In this case the influence of RESP on RR would be direct without the involvement of SBP, and a difference (delta value) between bi- and trivariate coupling index would be low. Secondly, a finding that the causal coupling index calculated through trivariate analysis is markedly lower than the index calculated through bivariate analysis indicates that RESP provides no further information explaining the RR oscillations if we already know the SBP oscillations. In this second case the influence of RESP on RR would be indirect and mediated mostly by baroreflex pathway $\mathrm{RESP} \rightarrow \mathrm{SBP} \rightarrow \mathrm{RR}$ and the delta value would be high.

In the light of these considerations, the results of the present study lead us to propose the schematic models of cardiorespiratory interactions illustrating the importance of direct/indirect pathways in the generation of RSA (Fig. 2). These models are inspired by the findings described in the following. (a)

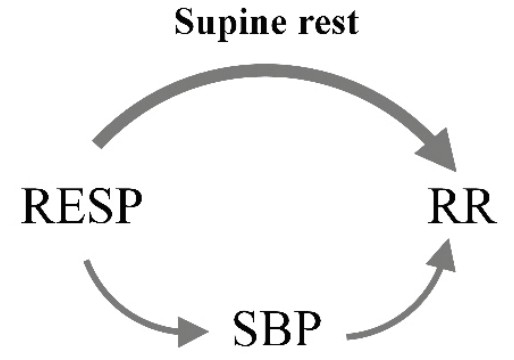

(b)

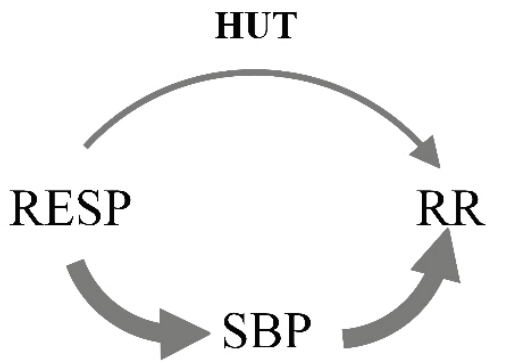

(c)

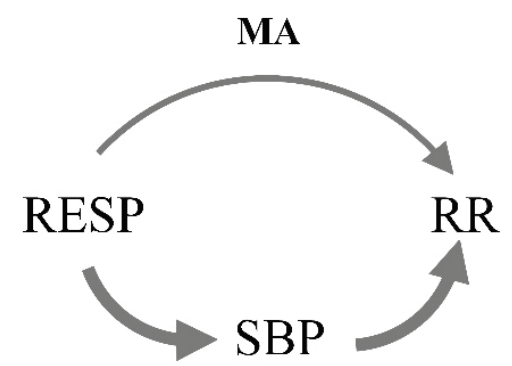

Fig. 2. Proposed models of RESP $\rightarrow$ RR interactions during supine rest (a), head-up tilt - HUT (b), and mental arithmetics - MA (c). The thickness of arrow reflects the strength of the causal coupling in the given direction. At rest, direct interaction between RESP and RR dominates reflecting central mechanism. During both challenges (HUT and MA), the importance of indirect - baroreflex based mechanism becomes more important. The strength of baroreflex mechanism is higher during HUT compared to MA.

Our results indicate that the overall strength of the influence from respiration to heart rate (bivariate $\mathrm{RESP} \rightarrow \mathrm{RR}$ coupling index) is stable across conditions. When analyzing the RESP - RR coupling in a trivariate way, the coupling indexes decreased to approximately half of the values observed for bivariate analysis. This indicates that both regulatory pathways, the one related to blood pressure oscillations (i.e. the baroreflex mechanism) and that independent on blood pressure oscillations (i.e. the mostly central mechanism), act as mechanisms involved in the origin of RSA. Further analysis considering the difference between bivariate and trivariate indexes (delta values) revealed that during supine rest and supine recovery the indirectionality of the interaction from RESP to RR was generally low. This means that during supine rest the direct influence $\mathrm{RESP} \rightarrow \mathrm{RR}$ (central mechanism) dominates over the indirect connection $\mathrm{RESP} \rightarrow \mathrm{SBP} \rightarrow \mathrm{RR}$ (peripheral mechanism - baroreflex mediated) (Fig. 2a). These results are in agreement with previous studies (Faes et al. 2011, Porta et al. 2012a, Porta et al. 2012b) where a stronger influence of the central mechanism during supine rest was also observed.

The impact of gravity during orthostasis (HUT) causes venous pooling of the blood in the lower portion of the body and a decrease of arterial blood pressure sensed by high-pressure baroreceptors. The responses include cardiac parasympathetic withdrawal and 
increased sympathetic activity to the heart and vasculature (Cooke et al. 1999). We extend previous observations on the decrease of the RSA magnitude during HUT (Laitinen et al. 2004, Patel et al. 2016) showing that during HUT the contribution of the baroreflex to RSA generation is enhanced, as reflected by the significantly increased delta values (indirectionality index) (Fig. 2c). Our observations are in concert with previous findings based on the analysis of cardiovascular oscillations (Faes et al. 2011, Porta et al. 2012b) that suggested a dominant central mechanism at supine rest and an increased involvement of the baroreflex during orthostasis.

During cognitive load, parasympathetic withdrawal and sympathetic activation are presumably initiated by projections from the amygdala and occur without major changes in venous return. Thus, mental stress is accompanied by a decrease of parasympathetic activity and/or an increase of the sympathetic activity (Kuipers et al. 2008, Widjaja et al. 2015); in this condition, RSA magnitude is usually decreased (Allen and Crowell 1989, Bernardi et al. 2000, Herbert et al. 2010, Wetzel et al. 2006, Javorka et al. 2018). In addition to these previous findings, we observed that the information flow from RESP to RR was amplified as regards the indirect connection during MA, but the degree of indirectionality was significantly lower than that during HUT ( $\mathrm{P}=0.0255)$ (Fig. 2c). Our results indicate that the relative contribution of mechanisms behind RSA does not change uniformly with a shift in sympatho-vagal balance towards sympathetic nervous system dominance and is more state/condition specific with the higher involvement of baroreflex during orthostasis compared to mental effort.

It is well known, that variations in respiratory pattern (respiratory rate, tidal volume) leads to changes in RSA magnitude - an increase of length of respiratory cycle and/or volume lead to an increase in RSA amplitude independently of vagal tone (Elghozi et al. 1991, Schmitz et al. 1995, Larsen et al. 2010). Therefore, it is conceivable to assume that the importance of different mechanisms in the generation of RSA can also vary with the respiratory rate. In our study, the average respiratory rate was $16.3 \pm 3.3$ breaths per minute (bpm) during the supine rest, $15.6 \pm 3.0 \mathrm{bpm}$ during HUT, $16.8 \pm 2.8 \mathrm{bpm}$ during first supine recovery, 20.6 $\pm 3.7 \mathrm{bpm}$ during MA, and $16.7 \pm 2.9 \mathrm{bpm}$ during second supine recovery. Although the orthostasis related differences in the RSA generation mechanisms could not be ascribed to the small respiratory rate change, a significantly increased respiratory rate during MA could be associated with the observed changes in information domain indices. The association between respiratory pattern changes and RSA mechanisms requires further study.

The primary purpose of this study was to compare the contribution of baroreflex and non-baroreflex (most importantly central) mechanisms in the generation of RSA. The effect of autonomic nervous system activity change on the assessed measures was demonstrated. Since the algorithm employed to calculate coupling measures involved signal quantization, the coupling indices are mathematically independent on the signal magnitude - i.e. on the magnitude of RSA (Faes et al. 2011, Faes et al. 2012). Although we did not intend to introduce new measure(s) for the assessment of cardiovascular control, the coupling indices used in this study were sensitive to shifts in autonomic nervous system activity accompanying orthostatic and mental challenges. Considering their mathematical independence and sensitivity to changed autonomic activity, the coupling indices could complement the existing autonomic nervous system activity indices in future physiological and clinical studies.

Taken together, results of our study confirmed on a larger sample of subjects $(n=78)$ the observations of few previous studies and extended the findings to healthy adolescents. In addition, our study included also cognitive load as a physiological challenge to demonstrate its partially different effect on the RSA mechanisms as compared to supine rest and orthostasis. We conclude that both basic mechanisms take part in RSA generation in the intact cardiorespiratory control of human subjects. The central mechanism is more important during rest. On the contrary, during orthostatic and mental challenges the baroreflex based peripheral mechanism becomes more important.

\section{Conflict of Interest}

There is no conflict of interest.

\section{Acknowledgements}

The study was supported by grants APVV-0235-12, VEGA 1/0117/17, UK/108/2018 and project "Biomedical Centre Martin” ITMS code: 26220220187 , the project is co-financed from EU sources. 


\section{References}

AINSWORTH DM, SMITH CA, JOHNSON BD, EICKER SW, HENDERSON KS, DEMPSEY JA: Vagal contributions to respiratory muscle activity during eupnea in the awake dog. $J$ Appl Physiol 72: 1355-1361, 1992.

ALLEN MT, CROWELL MD: Patterns of autonomic response during laboratory stressors. Psychophysiology 26: 603-614, 1989.

ANREP GV, PASCUAL W, ROSSLER R: Respiratory variations of the heart rate. I. The reflex mechanism of the respiratory arrhythmia. Proc Biol Sci 119: 191-217, 1936.

BEN-TAL A, SHAMAILOV SS, PATON JFR: Central regulation of heart rate and the appearance of respiratory sinus arrhythmia: New insights from mathematical modeling. Math Biosci 255: 71-82, 2014.

BERNARDI L, WDOWCZYK-SZULC J, VALENTI C, CASTOLDI S, PASSINO C, SPADACINI G, SLEIGHT P: Effects of controlled breathing, mental activity and mental stress with or without verbalization on heart rate variability. J Am Coll Cardiol 35: 1462-1469, 2000.

BERNTSON GG, CACIOPPO JT, QUIGLEY KS: Respiratory sinus arrhythmia: Autonomic origins, physiological mechanisms, and psychophysiological implications. Psychophysiology 30: 183-196, 1993.

COOKE WH, HOAG JB, CROSSMAN AA, KUUSELA TA, TAHVANAINEN KU, ECKBERG DL: Human responses to upright tilt: a window on central autonomic integration. $J$ Physiol 517: 617-628, 1999.

DRAGHICI AE, TAYLOR JA: The physiological basis and measurement of heart rate variability in humans. $J$ Physiol Anthropol 35: 22, 2016.

ELGHOZI JL, LAUDE D, GIRARD A: Effects of respiration on blood pressure and heart rate variability in humans. Clin Exp Pharmacol Physiol 18: 735-742, 1991.

ECKBERG DL: Point: Counterpoint: Respiratory sinus arrhythmia is due to a central mechanism vs. respiratory sinus arrhythmia is due to the baroreflex mechanism. J Appl Physiol 106: 1740-1742, 2009.

FAES L, NOLLO G, PORTA A: Information domain approach to the investigation of cardio-vascular, cardiopulmonary, and vasculo-pulmonary causal couplings. Front Physiol 2: 80, 2011.

FAES L, NOLLO G, PORTA A: Non-uniform multivariate embedding to assess the information transfer in cardiovascular and cardiorespiratory variability series. Comput Biol Med 42: 290-297, 2012.

FEI L, COPIE X, MALIK M, CAMM AJ: Short- and long-term assessment of heart rate variability for risk stratification after acute myocardial infarction. Am J Cardiol 77: 681-684, 1996a.

FEI L, KEELING PJ, SADOUL N, COPIE X, MALIK M, MCKENNA WJ, CAMM AJ: Decreased heart rate variability in patients with congestive heart failure and chronotropic incompetence. Pacing Clin Electrophysiol 19: 477-483, 1996b.

GROSSMAN P, TAYLOR EW: Toward understanding respiratory sinus arrhythmia: Relations to cardiac vagal tone, evolution and biobehavioral functions. Biol Psychol 74: 263-285, 2007.

HERBERT BM, POLLATOS O, FLOR H, ENCK P, SCHANDRY R: Cardiac awareness and autonomic cardiac reactivity during emotional picture viewing and mental stress. Psychophysiology 47: 342-354, 2010.

JAVORKA M, KROHOVA J, CZIPPELOVA B, TURIANIKOVA Z, LAZAROVA Z, JAVORKA K, FAES L: Basic cardiovascular variability signals: mutual directed interactions explored in the information domain. Physiol Meas 38: 877-894, 2017.

JAVORKA M, EL-HAMAD F, CZIPPELOVA B, TURIANIKOVA Z, KROHOVA J, LAZAROVA Z, BAUMERT M: Role of respiration in the cardiovascular response to orthostatic and mental stress. Am J Physiol Regul Integr Comp Physiol 314: R761-R769, 2018.

KUIPERS NT, SAUDER CL, CARTER JR, RAY CA: Neurovascular responses to mental stress in the supine and upright postures. $J$ Appl Physiol 104: 1129-1136, 2008.

LAITINEN T, NISKANEN L, GEELEN G, LÄNSIMIES E, HARTIKAINEN J: Age dependency of cardiovascular autonomic responses to head-up tilt in healthy subjects. $J$ Appl Physiol 96: 2333-2340, 2004.

LARSEN PD, TZENG YC, SIN PYW, GALLETLY DC: Respiratory sinus arrhythmia in conscious humans during spontaneous respiration. Respir Physiol Neurobiol 174: 111-118, 2010. 
LUDWIG C: Beiträge zur kenntniss des einflusses der respirationbewegungen auf den blutlauf im Aortensysteme. (In German) Arch Anat Physiol 13: 242-302, 1847.

MORTOLA JP, MARGHESCU D, SIEGRIST-JOHNSTONE R: Thinking about breathing: Effects on respiratory sinus arrhythmia. Respir Physiol Neurobiol 223: 28-36, 2016.

NOLLO G, FAES L, PORTA A, PELLEGRINI B, RAVELLI F, DEL GRECO M, DISERTORI M, ANTOLINI R: Evidence of unbalanced regulatory mechanism of heart rate and systolic pressure after acute myocardial infarction. Am J Physiol Heart Circ Physiol 283: H1200-H1207, 2002.

NOLLO G, FAES L, PORTA A, ANTOLINI R, RAVELLI F: Exploring directionality in spontaneous heart period and systolic pressure variability interactions in humans: implications in the evaluation of baroreflex gain. Am J Physiol Heart Circ Physiol 288: H1777-H1785, 2005.

PATEL K, RÖSSLER A, LACKNER HK, TROZIC I, LAING C, LORR D, GREEN DA, HINGHOFER-SZALKAY H, GOSWAMI N: Effect of postural changes on cardiovascular parameters across gender. Medicine 95: e4149, 2016.

PIEPOLI M, SLEIGHT P, LEUZZI S, VALLE F, SPADACINI G, PASSINO C, JOHNSTON J, BERNARDI L: Origin of respiratory sinus arrhythmia in conscious humans. An important role for arterial carotid baroreceptors. Circulation 95: 1813-1821, 1997.

PORTA A, CATAI AM, TAKAHASHI ACM, MAGAGNIN V, BASSANI T, TOBALDINI E, VAN DE BORNE P, MONTANO N: Causal relationships between heart period and systolic arterial pressure during graded head-up tilt. Am J Physiol Regul Integr Comp Physiol 300: R378-R386, 2011.

PORTA A, BASSANI T, BARI V, PINNA GD, MAESTRI R, GUZZETTI S: Accounting for respiration is necessary to reliably infer granger causality from cardiovascular variability series. IEEE Trans Biomed Eng 59: 832-841, 2012a.

PORTA A, BASSANI T, BARI V, TOBALDINI E, TAKAHASHI ACM, CATAI AM, MONTANO N: Model-based assessment of baroreflex and cardiopulmonary couplings during graded head-up tilt. Comput Biol Med 42: 298-305, 2012b.

SAUL JP, BERGER RD, ALBRECHT P, STEIN SP, CHEN MH, COHEN RJ: Transfer function analysis of the circulation: Unique insights into cardiovascular regulation. Am J Physiol 261: H1231-H1245, 1991.

SCHMITZ JM, CLAUS D, NEUNDÖRFER B, HANDWERKER HO: Comparison of different algorithms for evaluation of respiratory sinus arrhytmia: cross-correlation function histogram analysis and regression analysis. Physiol Res 44: 197-203, 1995.

SCHÄFER C, ROSENBLUM MG, KURTHS J, ABEL HH: Heartbeat synchronized with ventilation. Nature 392: 239-240, 1998.

TAHA BH, SIMON PM, DEMPSEY JA, SKATRUD JB, IBER C: Respiratory sinus arrhythmia in humans: an obligatory role for vagal feedback from the lungs. $J$ Appl Physiol 78: 638-645, 1995.

ZHANG R, IWASAKI K, ZUCKERMAN JH, BEHBEHANI K, CRANDALL CG, LEVINE BD: Mechanism of blood pressure and R-R variability: insights from ganglion blockade in humans. J Physiol 543: 337-348, 2002.

WETZEL JM, QUIGLEY KS, MORELL J, EVES E, BACKS RW: Cardiovascular measures of attention to illusory and nonillusory visual stimuli. $J$ Psychophysiol 20: 276-285, 2006.

WIDJAJA D, MONTALTO A, VLEMINCX E, MARINAZZO D, VAN HUFFEL S, FAES L: Cardiorespiratory information dynamics during mental arithmetic and sustained attention. PLoS One 10: e0129112, 2015. 